\title{
RESTORATION OF DETERMINISTIC AND INTERFERENCE DISTORTED SIGNALS AND IMAGES WITH USE OF THE GENERALIZED SPECTRA BASED ON ORTHOGONAL POLYNOMIALS AND FUNCTIONS
}

Restoration algorithms of signals and images on the basis of their generalized spectra in bases of orthogonal polynomials and functions at absence and presence of random distortions have been examined. It is shown that in absence of hindrances the number of coefficients of the generalized spectrum of a restored signal (image) is determined by the desired approximation error at use of one or another metrics of functional space. If hindrances take place then there is an optimum number of coefficients of the generalized spectrum for signal (image) restoration. Working data of the proposed algorithms for various types of useful signals have been illustrated.

Keywords: Signal and image restoration, generalized signal spectrum, orthogonal polynomials, Gauss quadrature formulas, approximation mean square error, maximum likelihood method, incomplete signal reception.

\section{Introduction}

One of the methods of increasing throughput of multimedia data transmission, receiving and processing systems is optimization and improvement of coding (decoding) procedures of a signal source and redundancy reduction. At present two essentially different approaches for information compression of signals and images are used: 1) lossless compression; 2) controllable lossy compression [1,2]. The first approach is based on principles of differential pulse code modulation, Huffman entropy coding, Lempel-Ziv-Welch method of repetitive chapter search on samples, as well as some others and it cannot provide essential compression of a data flow. More expedient is the coding procedure of a signal source with controlled losses. Within the scope of this approach various linear orthogonal transforms are applied: a) discrete cosine transform and its updatings; b) wavelet-transform; c) discontinuous piecewise constant function basis expansion (such as Walsh, Haar, S-transformation, etc.), however all of them are not flawless [2]. It causes need of new basic function search.

It is obvious that new algorithms of signal and image compression should be optimized not only in respect of computational burden reduction, complexity of hardware implementation but also in statistical sense taking into account probabilistic nature of hindrances, messages and performance measures. This problem is so severe under image transmission and processing as images have sufficiently great information capacity. In the present work it is shown that, as in [3, 4], the application of orthogonal polynomials or functions connected with them allows to receive effective, practically realizable procedures of signal and image restoration including presence of random distortions.

\section{Signal and image restoration without noise influence}

As systems of orthogonal polynomials (functions), the following polynomials (functions) can be chosen [5]

Legendre polynomials

$$
P_{n}(x)=\frac{1}{2^{n} n !} \frac{d^{n}}{d x^{n}}\left(x^{2}-1\right)^{n},
$$

Chebyshev polynomials of the first kind

$$
T_{n}(x)=\frac{(-2)^{n} n !}{(2 n) !} \sqrt{1-x^{2}} \frac{d^{n}}{d x^{n}}\left(\sqrt{1-x^{2}}\right)^{2 n-1},
$$

Hermite polynomials

$$
H_{n}(x)=(-1)^{n} \exp \left(x^{2}\right) \frac{d^{n}}{d x^{n}} \exp \left(-x^{2}\right),
$$

Hermite functions

$$
\varphi_{n}(x)=\frac{1}{\sqrt{2^{n} n ! \sqrt{\pi}}} H_{n}(x) \exp \left(-\frac{x^{2}}{2}\right),
$$

etc. In Eqs. (1)-(4) $n$ is nonnegative integer.

\footnotetext{
* Oleg V. Chernoyarov ${ }^{1}$, Martin Breznan ${ }^{2}$, Alexander V. Terekhov ${ }^{1}$

${ }^{1}$ National Research University “Moscow Power Engineering Institute”, Moscow, Russia, E-mail: o_v_ch@mail.ru

${ }^{2}$ Department of Telecommunications and Multimedia, University of Zilina, Slovakia
} 
The generalized spectra $C_{n}^{P}, C_{n}^{T}, C_{n}^{H}, C^{\prime H}$ of the one-dimensional signals $s(x)$ specified on the interval $x \in[a, b]$ when using (1)-(4) can be accordingly found as

$$
\begin{aligned}
& C_{n}^{P}=\frac{2 n+1}{2} \int_{-1}^{1} s\left(\frac{b-a}{2} x+\frac{a+b}{2}\right) P_{n}(x) d x, \\
& C_{n}^{T}=\frac{2}{\pi \alpha_{n}} \int_{-\pi / 2}^{\pi / 2} s\left[\frac{b-a}{2} \sin (x)+\frac{a+b}{2}\right] . \\
& \cdot T_{n}[\sin (x)] d x, \quad \alpha_{n}\left\{\begin{array}{l}
2 . n=0 ; \\
1, n \neq 0 ;
\end{array}\right. \\
& C_{n}^{H}=\frac{1}{2^{n} n ! \sqrt{\pi}} \int_{-A}^{A} s\left(\frac{b-a}{2 A} x+\frac{a+b}{2}\right) . \\
& H_{n}(x) \exp \left(-x^{2} d x\right), \\
& C^{\prime H}=\int_{-A}^{A} s\left(\frac{b-a}{2 A} x+\frac{a+b}{2}\right) \varphi_{n}(x) d x, \\
& A>>1
\end{aligned}
$$

Then in absence of hindrances the signal restoration operation on the generalized spectrum will be written in the form

$$
s(x)=\sum_{n=0}^{\infty} C_{n} \Psi_{n}\left(\gamma \frac{2 x-a-b}{b-a}\right),
$$

where $\left\{\Psi_{n}(x)\right\}$ is one of the orthogonal function systems (1)-(4), $\left\{\mathrm{C}_{n}\right]$ is generalized spectrum (5)-(7) corresponding to the basis $\left\{\Psi_{n}(x)\right\}, \gamma=A$ for Hermite polynomials (functions) (7) and $\gamma=1$ for Legendre and Chebyshev polynomials. Generalization of formulas (5)-(8) on a two-dimensional case is obvious.

As the analysis showed, expansion coefficients (5)-(7) decrease rapidly with increasing $\mathrm{n}$ for both continuous (differentiable and nondifferentiable) and discontinuous signals. However for practical applications the calculation of the generalized spectra according to the formulas (5)-(7) should be made numerically in most cases. Thereupon it is of interest to use the expansion procedures which minimize computing expenses. One of the possible ways for creating such procedures is the use of Gauss quadrature formulas of the maximum degree of precision [6]:

$$
\int s(x) \rho(x) d x=\sum_{k=0}^{N-1} \lambda_{k} s\left(x_{k}\right)
$$

Here $x_{k}$ are zeros of a polynomial of $\mathrm{N}$-th order, $\rho_{x}$ is a weight function for polynomial orthogonality [7], $\lambda_{k}$ are Christoffel numbers, and integration is conducted on an orthogonality interval of polynomials used in expancion. Special cases of Eq. (9) for polynomials (1)-(4) have the appearance:

1. Gauss-Legendre formula:

$$
\int_{-1}^{1} s(x) d x=\sum_{k=0}^{N-1} \lambda_{k} s\left(x_{k}\right),
$$

where $x_{k}$ are zeros of equation $P_{N}(x)=0, \lambda_{k}=2 /\left(1-x_{k}^{2}\left[P_{N}^{\prime}\left(x_{k}\right]^{2}\right.\right.$.

2. Mehler (Gauss-Chebyshev) formula:

$$
\int_{-1}^{1} \frac{s(x)}{\sqrt{1-x^{2}}} d x=\sum_{k=0}^{N-1} \lambda_{k} s\left(x_{k}\right),
$$

where $x_{k}=\cos [\pi(2 k+1) / 2 N]$ are zeros of Chebyshev polyno$\operatorname{mial} T_{N}(x), \lambda_{k}=\pi / N$.

3. Gauss-Hermite formula:

$$
\int_{-\infty}^{\infty} s(x) \exp \left(-x^{2}\right) d x=\sum_{k=0}^{N-1} \lambda_{k} s\left(x_{k}\right),
$$

where $x_{k}$ are zeros of equation $H_{N}(x)=0, \lambda_{k}=2^{N+1} N ! \sqrt{\pi /}$ $/\left[H^{\prime}{ }_{N}\left(x_{k}\right)\right]^{2}$.

Using (10)-(12) the formulas (5)-(7) for the generalized signal spectra can be rewritten as

$$
\begin{aligned}
& C_{n}^{P}=\sum_{k=0}^{N-1} \frac{2 n+1}{\left(1-x_{k}^{2}\right)\left[P_{N}^{\prime}\left(x_{k}\right)\right]^{2}} . \\
& s\left(\frac{b-a}{2} x_{k}+\frac{a+b}{2}\right) P_{n}\left(x_{k}\right), \\
& C_{n}^{T}=\frac{2}{N \alpha_{n}} \sum_{k=0}^{N-1} s\left[\frac{b-a}{2} \cos \left(\frac{\pi(2 k+1)}{2 N}\right)+\frac{a+b}{2}\right] . \\
& \cdot \cos \left[\frac{\pi n(2 k+1)}{2 N}\right], \\
& C_{n}^{H}=\frac{2^{N-n+1} N !}{n !} \sum_{k=0}^{N-1} \frac{1}{\left[H_{N}^{\prime}\left(x_{k}\right)\right]^{2}} . \\
& \cdot s\left(\frac{b-a}{2} x_{k}+\frac{a+b}{2}\right) H_{n}\left(x_{k}\right), \\
& C^{\prime H}=N ! \sqrt{\frac{2^{N-n+3} \sqrt{\pi}}{n-1}} \sum_{k=0}^{N} \frac{1}{\left[H_{N}^{\prime}\left(x_{k}\right)\right]^{2}} . \\
& \cdot s\left(x_{k} \sqrt{2}\right) H_{n}\left(x_{k} \sqrt{2}\right) .
\end{aligned}
$$

On the basis of Eqs. (13)-(16) and similar expressions the study of spectra for various signal models (differentiable, continuous nondifferentiable and discontinuous) was conducted. As an example in Table 1, coefficients $C_{n}^{P}, C_{n}^{T}, C_{n}^{H}, C^{\prime H}{ }_{n}, 0 \leq n \leq 20$ calculated according to formulas (13)-(16) for typical functions $s(x)=\exp \left(-x^{2}\right)$ (differentiable); $s(x)=1-|x|,-1 \leq x \leq 1$ (continuous nondifferentiable); $s(x)=\operatorname{sgn}(x)$, where $\operatorname{sgn}(x)=1$ if $x \geq 0$ and $\operatorname{sgn}(x)=-1$ if $x<0$ (discontinuous) are resulted. In that table the values of mean square error $\delta=\delta(N)$ [8]

$$
\delta=\frac{1}{b-a} \int_{a}^{b}\left[s(x)-\sum_{n=0}^{N} C_{n} \Psi(x)\right]^{2} d x
$$

of signal approximation on the basis of the generalized spectra calculated at $a=-1, b=1$ (orthogonality interval of polynomials (1), (2)) and various $\mathrm{N}$ are specified. Here $C_{n}$ is one of the coefficients $C_{n}^{P}, C_{n}^{T}, C_{n}^{H}, C^{\prime}{ }_{n}^{H}(13)-(16)$, and $\Psi_{n}(x)$ is corresponding polynomial (function) of $\mathrm{n}$-th order (1)-(4).

From the Table 1 and conducted analysis including other orthogonal bases there follows that for differentiable and contin- 
uous nondifferentiable signals the construction of the generalized spectra on the basis of Hermite polynomials (3) or functions (4) appears the most effective. Really, coefficients $C_{n}^{H}(15), C^{\prime}{ }_{n}^{H}(16)$ converge rapidly enough to zero with increasing $n$ so mean square error (17) becomes relatively small at $\mathrm{N}<5-6$. The use of Legendre and Chebyshev polynomials can provide smaller extent of $\delta$ (17) in comparison with Hermite polynomials (functions). However sets $\left\{C_{n}^{P}\right\},\left\{C_{n}^{T}\right\}$ can essentially exceed sets $\left\{C_{n}^{H}\right\},\left\{C^{\prime}{ }_{n}^{H}\right\}$ by number of significant coefficients.

For discontinuous signals the Hermite polynomials (functions) also provide the least number of significant coefficients of the generalized spectrum. However approximation mean square error (17) received at their use reaches relatively large values. In this case the construction of the generalized spectrum on the basis of Chebyshev polynomials can appear to be expedient. Really, at loss by number of coefficients in 2 times approximately in comparison with Hermite series the accuracy gain of the signal approximation estimated according to (17) can reach 3-3.5 times. Also it should be noted that expansion coefficients calculated with the use of quadrature formulas of the maximum degree of precision which have an order less than $10^{-4}$ can be omitted at construction of the generalized spectrum of an analyzed signal. Besides, rate of convergence for series (8) on orthogonal polynomials can be more rapid generally than for basis of trigonometric functions and discrete cosine transformation [2].

\section{Signal and image restoration in the presence of random distortions}

As it has been established in the section 2 at restoration of the deterministic signals and images by their generalized spectra the number of restored signal (image) modes (coefficients of the generalized spectrum) is determined by the desired approximation error at use of one or another metrics of functional space. It will be shown below that the account of fluctuating noises actually existing together with a useful signal leads to occurrence of optimum number of modes which should be used for image restoration.

So, let the field

$$
\xi(r)=s_{M}(r)+\eta(r), \quad r=(x, y)
$$

be received to the optical-electron converter's input. Here $s_{M}$ is observable useful signal for which the form and other characteristics can be apriori unknown, $\eta(r)$ is hindrance. Let us suppose that the useful signal $s_{M}(r)$ can be presented as series

$$
s_{M}(r)=s_{M}(x, y)=\sum_{m=0}^{M} \sum_{n=0}^{M} c_{m n} \varphi_{m}(x) \varphi_{n}(y)
$$

in one or another orthonormal function system $\left\{\varphi_{m n}(x)\right\}$. Here $C_{m n}=\int_{\Omega} s_{M}(x, y) \varphi_{m}(\mathrm{x}) \varphi_{n}(\mathrm{y})$ are expansion coefficients (generalized signal spectrum) and $\Omega$ is area of orthogonality of basis $\left\{\varphi_{m}(x) \varphi_{m}(y)\right\}$.
Models of hindrances at optical and optoelectronic processing of the information are in detail considered in $[9,10]$. If a quantum character of weak optical signals is not taken into consideration and signal $s_{M}(r)$ is assumed as intensive enough then it is possible often to believe that a hindrance $\eta(r)$ is Gaussian random field. In addition, if processing algorithms do not consider thin difference of spatial spectra of a useful signal and hindrance but focus the attention on the analysis of generalized spectrum's modes of the field $s_{M}(r)$ then hindrance $\eta(r)$ may be approximated by Gaussian white noise with a correlation matrix of a kind $K_{\eta}\left(r_{1}, r_{2}\right)=$ $=\left(N_{0} / 2\right) \delta\left(r_{2}-r_{1}\right)$. Here $N_{0} / 2$ is spectral density (intensity) of white noise. The model $\eta(r)$ as white noise is quite proved if hindrance sources are wideband processes in electronic devices [11] and will be used by us further.

On observable realization $\xi(r)$ and the available prior information it is necessary to restore an original signal $s_{M}(r)$ in an optimal way.

In the task of signal (image) restoration at the presence of random hindrances the vector $\vec{C}=\left(C_{0}, \ldots, C_{M}\right)$ is unknown and cannot be calculated directly as in (5)-(7). Therefore in the processing operations it is necessary to receive an estimate of this vector. At synthesis of estimation algorithm we will use a maximum likelihood method. Without loss of generality of obtained results for simplification of mathematical calculations we will believe a signal $s_{M}(r)$ as one-dimensional in the sequel, i.e. $s_{M}(r)=s_{M}(x)$. Then following [12] a logarithm of functional of likelihood ratio $L$ as function of current values of all unknown coefficients $C_{n}, 0 \leq n \leq M$ can be written down in the form:

$$
L(\vec{C})=\frac{1}{N_{0}}\left[2 \int_{\Omega} \xi(x) s_{M}(x, \vec{C}) d x-\int_{\Omega} s_{M}^{2}(x, \vec{C}) d x\right] .
$$

Using the representation of a useful signal (18) in Eq. (19) we have

$$
L(\vec{C})=\frac{1}{N_{0}} \sum_{n=0}^{M}\left(2 X_{n} C_{n}-C_{n}^{2}\right) .
$$

Here $X_{n}=\int_{\Omega} \xi(x) \varphi_{n}(x) d x$. As maximum likelihood estimates

(MLEs) $\widetilde{\vec{C}}=\left(\widetilde{C}_{0}, \ldots, \widetilde{C}_{M}\right)$ of measured parameters the position of the greatest maximum of solving statistics (20) is taken:

$$
\tilde{\vec{C}}=\underset{\vec{C}}{\arg \sup } L(\vec{C}) .
$$

Estimates $\widetilde{C}_{n}, 0 \leq n \leq M$ can be found from the decision of the likelihood equations

$$
\begin{aligned}
& \partial L(\vec{C}) / \partial C_{0}=0, \partial L(\vec{C}) / \partial C_{1}=0, \ldots, \\
& \partial L(\vec{C}) / \partial C_{M}=0 .
\end{aligned}
$$

Substituting (20) in (21) and carrying out simple transformations we come to MLEs of a kind

$$
\vec{C}_{n}=X_{n}=\int_{\Omega} \xi(x) \varphi_{n}(x) d x, 0 \leq n \leq M .
$$




\begin{tabular}{|c|c|c|c|c|c|c|c|c|c|c|c|c|c|c|c|c|c|c|c|c|c|c|c|c|}
\hline 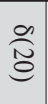 & $\stackrel{\substack{0 \\
\bar{v}}}{=}$ & $\frac{\infty}{\hat{c}}$ & $\stackrel{\infty}{(\vec{v}}$ & $\tilde{\sigma}$ & 5 & $\vec{\infty}$ & $\Xi$ & $\bar{a}$ & $\vec{u}$ & $\mp$ & $\bar{\omega}$ & $\bar{\sim}$ & $=$ & $\overrightarrow{0}$ & 0 & $\infty$ & $\sim$ & $a$ & $\backsim$ & $\rightarrow u$ & $N$ & - & 0 & $=$ \\
\hline$\stackrel{*}{*}$ & $\mid \begin{array}{l}\vec{N} \\
\stackrel{*}{*} \\
\overrightarrow{O_{i}}\end{array}$ & $\begin{array}{l}\vec{*} \\
\vec{*} \\
\dot{P}_{\infty}\end{array}$ & 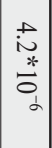 & 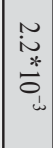 & $\stackrel{\substack{* \\
\stackrel{*}{*}}}{\stackrel{*}{i}}$ & $\begin{array}{l}\breve{u} \\
\vec{w} \\
\overrightarrow{0} \\
+\end{array}$ & 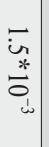 & $\begin{array}{l}b \\
\dot{b} \\
* \\
0 \\
0 \\
+\end{array}$ & $\begin{array}{c}\frac{1}{\vec{u}} \\
\vec{u} \\
\dot{u} \\
\dot{\omega}\end{array}$ & $\begin{array}{l}1 \\
0 \\
0 \\
\ddot{1} \\
0_{1} \\
+\end{array}$ & $\begin{array}{l}\stackrel{\dot{\sim}}{-} \\
\stackrel{*}{\sigma} \\
\stackrel{\omega}{\omega}\end{array}$ & 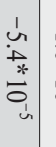 & $\begin{array}{l}\dot{\vec{H}} \\
\dot{\overrightarrow{0}} \\
\dot{\vec{b}}\end{array}$ & 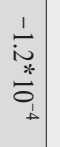 & 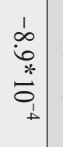 & 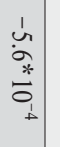 & 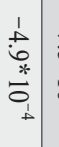 & & 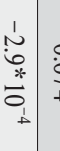 & & 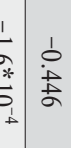 & 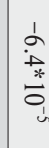 & 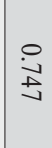 & $=0$ \\
\hline 0 & 0 & 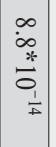 & $\left|\begin{array}{c}u \\
\tilde{u} \\
\tilde{0}\end{array}\right|$ & 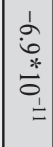 & 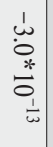 & $\begin{array}{c}\frac{w}{3} \\
\stackrel{*}{0} \\
\frac{1}{N} \\
\end{array}$ & 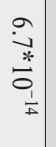 & $\begin{array}{c}\vec{f} \\
\stackrel{*}{*} \\
\overrightarrow{0} \\
\stackrel{1}{0}\end{array}$ & 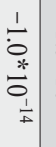 & $\begin{array}{l}\frac{1}{\dot{U}_{1}} \\
\overrightarrow{\sigma_{\infty}} \\
\dot{\infty}\end{array}$ & 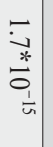 & 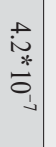 & $\begin{array}{l}\frac{1}{i} \\
\stackrel{*}{0} \\
\frac{1}{n}\end{array}$ & 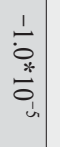 & 0 & $\begin{array}{l}\tilde{y} \\
\tilde{0} \\
\tilde{0} \\
0 \\
1\end{array}$ & o & 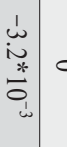 & ○ & & 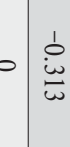 & 0 & 方 & $\approx$ \\
\hline & $\mid \begin{array}{l}\overrightarrow{\vec{*}} \\
\overrightarrow{\sigma_{\vec{b}}}\end{array}$ & $\mid$\begin{tabular}{l}
$\vec{*}$ \\
\multirow{2}{*}{}
\end{tabular} & $\stackrel{8}{*}$ & 0 & 。 & $\begin{array}{l}\frac{1}{\bar{*}} \\
\frac{*}{0} \\
\end{array}$ & 0 & $\begin{array}{l}0 \\
\dot{0} \\
\dot{0} \\
0 \\
\end{array}$ & 0 & do & 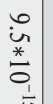 & $\ddot{\ddot{*}}$ & 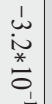 & $\begin{array}{l}\frac{1}{\infty} \\
\dot{0} \\
\frac{*}{0}\end{array}$ & 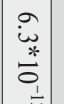 & $\begin{array}{l}\frac{1}{\sim} \\
\stackrel{*}{0} \\
\frac{0}{0}\end{array}$ & 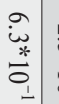 & 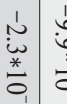 & 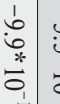 & 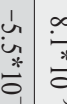 & 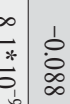 & & $\stackrel{0}{-}$ & 3 \\
\hline
\end{tabular}

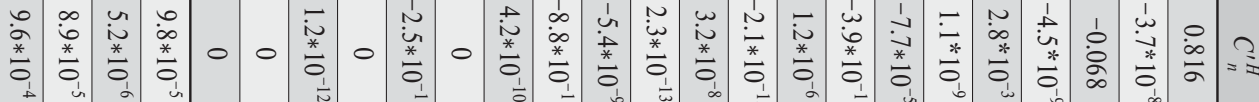

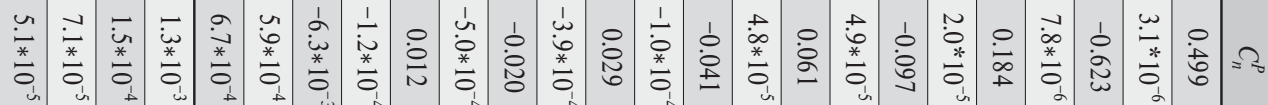

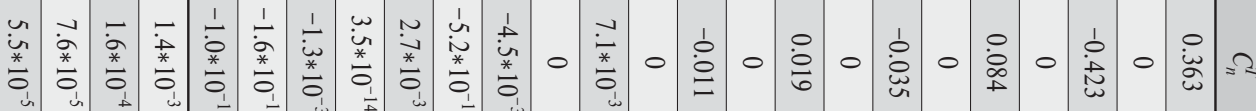

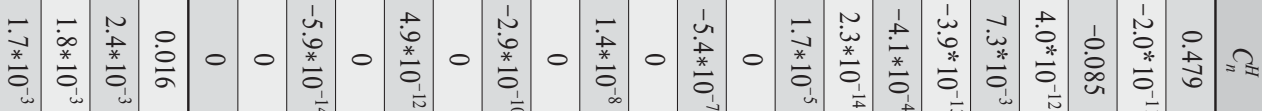

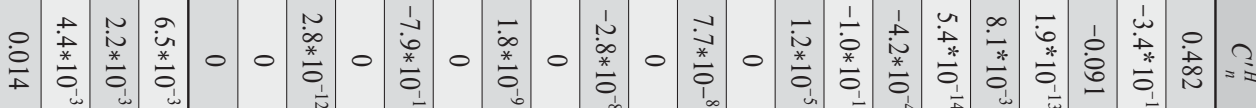

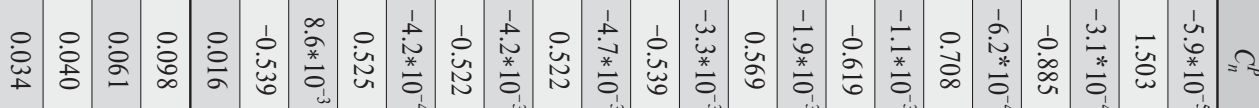

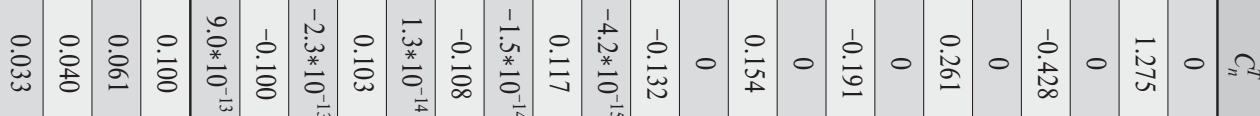

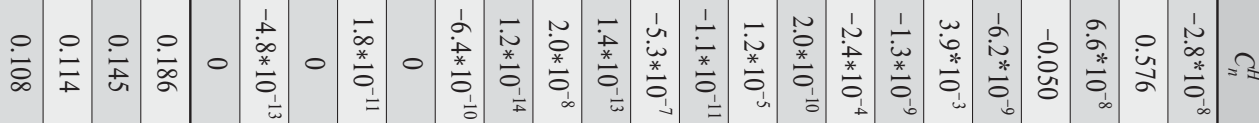

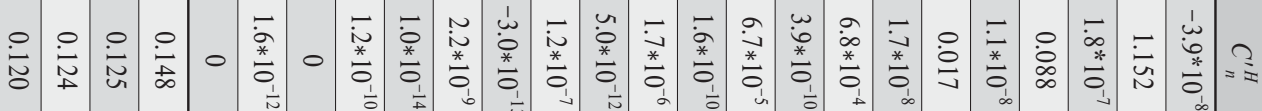


The found estimates (22) obviously allow to restore a useful signal $s_{M}(x)$ according to Eq. (18).

Let us find characteristics of MLEs (22). As MLEs' characteristics we will use their conditional biases (systematic errors) $b\left(\widetilde{C}_{n} \mid C_{n}\right)=\left\langle\widetilde{C}_{n}\right\rangle-C_{n}$ and variances (error mean squares) $V\left(\widetilde{C}_{n} \mid C_{n}\right)$ $=\left\langle\left(\widetilde{C}_{n}-C_{n}\right)^{2}\right\rangle$. Here angle brackets \langle\rangle designate averaging operation on all possible realizations $\xi(r)$. Carrying out direct averaging Eq. (22) we receive that the estimate $\widetilde{C}_{n}$ is conditionally unbiased: $b\left(\widetilde{C}_{n} \mid C_{n}\right)=0$ and its conditional variance has the appearance $V\left(\widetilde{C}_{n} \mid C_{n}\right)=N_{0} / 2$. Besides, by virtue of orthogonality of basis $\left\{\varphi_{n}(x)\right\}$ the estimates $\widetilde{C}_{n}, 0 \leq n \leq M$ are uncorrelated: $\left\langle\left(\widetilde{C}_{k}-C_{k}\right)\left(\widetilde{C}_{n}-\right.\right.$ $\left.\left.-C_{n}\right)\right\rangle=\left(N_{0} / 2\right) \int_{\Omega} \varphi_{k}(x) \varphi_{n}(x) d x=0, k \neq n$.

Let us assume that for restoration of a useful signal $s_{M}(x)$ $\mathrm{K}$ modes of expansion (22) are used. Then the restored signal $\tilde{s}_{K}(x)$ can be presented as follows

$$
\tilde{s}_{K}(x)=\sum_{n=0}^{K-1} \tilde{C}_{n} \varphi_{n}(x) .
$$

Define difference of the restored signal $\tilde{s}_{K}(x)(23)$ from original signal $s_{M}(x)(18)$ as $[12,13]$

$$
\begin{aligned}
& \langle\delta\rangle=\left\langle\int_{\Omega}\left[\tilde{s}_{K}(x)-s_{M}(x)\right]^{2} d x\right\rangle= \\
& =\sum_{n=0}^{K-1} V\left(\tilde{C}_{n} \mid C_{n}\right)+\sum_{n=K}^{M} C_{n}^{2}=\frac{N_{0}}{2}\left(K+\sum_{n=K}^{M} z_{n}^{2}\right) .
\end{aligned}
$$

Here $z_{n}^{2}=2 C_{n}^{2} / N_{0}$ is the signal-to-noise ratio (SNR) for a mode with an index n. According to Eq. (24) error $\langle\delta\rangle$ consists of two components. The first component is the fluctuating error increasing with $\mathrm{K}$ which is attributed to a deviation $\widetilde{C}_{n}$ from $C_{n}$. The second component is caused by energy of unrestored modes. It is obvious that it decreases with $\mathrm{K}$.

Let us denote $z_{\min }^{2}=\min \left(z_{\mathrm{K}}^{2}, z_{\mathrm{K}+1}^{2}, \ldots, z_{M}^{2}\right)$ and $z_{\max }^{2}=\max \left(z_{\mathrm{K}}^{2}\right.$, $\left.z_{\mathrm{K}+1}^{2}, \ldots, z_{M}^{2}\right)$ as minimum and maximum SNR in the residual mode signal expansion. Then from Eq. (24) following inequalities can be written down:

$$
\begin{aligned}
& (M+1) z_{\text {min }}^{2}+K\left(1-z_{\text {min }}^{2}\right)<N_{0}\langle\delta\rangle / 2< \\
& <(M+1) z_{\text {max }}^{2}+K\left(1-z_{\text {max }}^{2}\right) .
\end{aligned}
$$

Thus, the normalized error $\mu(K)=N_{0}\langle\delta\rangle / 2$ as function of integer variable $\mathrm{K}$ lies between two majorizing functions

$$
\begin{aligned}
& f_{1}(K)=(M+1) z_{\min }^{2}+K\left(1-z_{\min }^{2}\right), \\
& f_{2}(K)=(M+1) z_{\max }^{2}+K\left(1-z_{\max }^{2}\right) .
\end{aligned}
$$

In Fig. 1 the typical behavior of normalized error $\mu(K)$ for three cases is shown: a) $z_{\text {min }}^{2}<1, z_{\max }^{2}<1$ (Fig. 1a); b) $z_{\min }^{2}>1, z_{\max }^{2}>$ $>1$ (Fig. 1b); c) $z_{\min }^{2}<1, z_{\max }^{2}>1$ (Fig. 1c). Also here for comparison the dependences of functions $f_{1}(K)$ and $f_{2}(K)$ (25) are plotted. As test signal the signal $s_{M}(x)=\exp \left[-\alpha^{2}(x-\tau)^{2}\right]$ in basis of Hermite functions (4) was used. The generalized spectrum of such signal has the appearance [4]

$$
C_{n}=\frac{\alpha^{n}}{2^{n} n !\left(\alpha^{2}+1\right)^{(n+1) / 2}} \exp \left(-\frac{\alpha^{2} \tau^{2}}{\alpha^{2}+1}\right) H_{n}\left(\frac{\alpha \tau}{\sqrt{\alpha^{2}+1}}\right) .
$$

At construction of graphs it was taken $\alpha=1, \tau=2$ and first six modes of expansion $(M=5)$ were discounted. The noise spectral density for Figs. 1a-1c was accepted equal 1, 0.02 and 0.2 respectively.

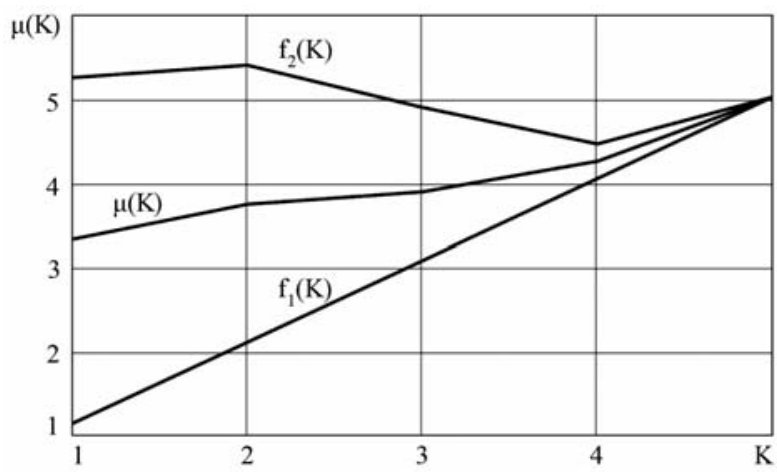

a)

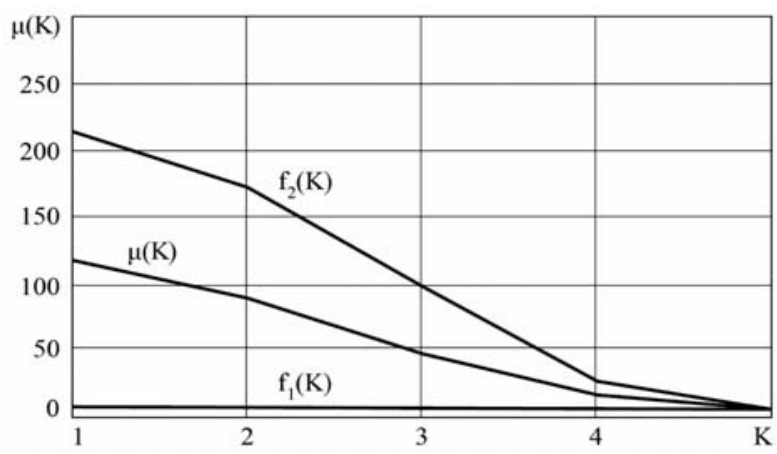

b)

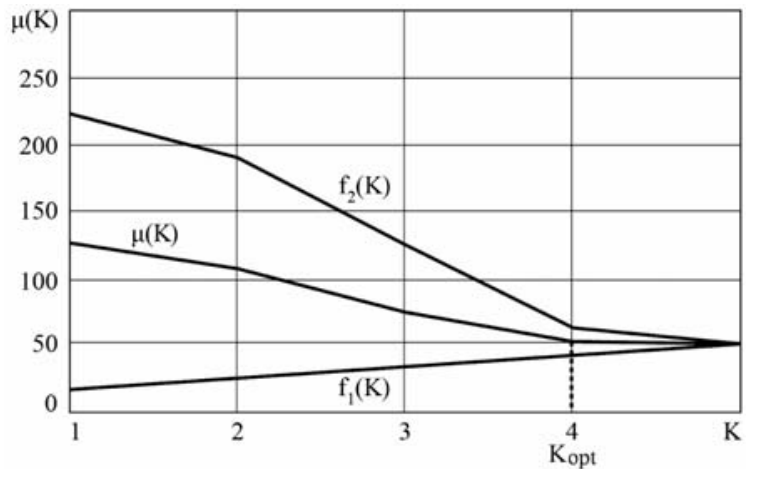

c)

Fig. 1 Normalized restoration error of a useful signal at various intensities of acting hindrances 
As follows from Fig. 1 under conditions $z_{\min }^{2}<1, z_{\max }^{2}<1$ the function $\mu(K)$ is monotonously increasing (i.e., with increasing number of processed modes the restoration error of a useful signal (24) also increases). If $z_{\min }^{2}>1, z_{\max }^{2}>1$ then the function $\mu(K)$ (and therefore the restoration error (24)) monotonously decreases with increasing number of processed modes. At last, in case of $z_{\min }^{2}<1, z_{\max }^{2}>1$ the behavior of function $\mu(K)$ becomes nonmonotonic. Really, having rewritten Eq. (24) as

$$
\mu(K)=M+1+\sum_{n=K}^{M}\left(z_{n}^{2}-1\right)
$$

it is possible to see that while condition $z_{n}^{2}>1$ holds summands under summation symbol (26) are positive and $\mu(K)$ decreases with increasing $\mathrm{K}$. However, with the beginning of fulfillment of the condition $z_{n}^{2}<1$ summands in Eq. (26) change a sign on opposite and the error $\mu(K)$ starts to increase. The optimum number of restored modes $K_{o p t}$ corresponding to the minimum restoration error $\mu(K)$ of a useful signal $s_{M}(x)$ is determined by the relation $z_{K}^{2}=1$ (or more exactly $z_{K}^{2}>1, z_{K+1}^{2}<1$ ) for the considered example $K_{\text {opt }}=4$ (Fig. 1c). Physically this is explained by the fact that under $z_{n}^{2}<1$ the fluctuating error connected with restoration of such modes is more than a deterministic component of the total error. Therefore, further expansion of signal components $\tilde{s}_{K}(x)$ becomes inexpedient.

Qualitative illustration of operation of the synthesized restoration algorithm (22), (23) at various types of the useful signal observed against white noise is shown in Fig. 2. Here the original useful signal $s_{M}(x)(M=\infty)$ is plotted by solid lines, the realization of the observed data $\xi(x)$ - by dashed lines and the restored signal $\tilde{s}_{K}(x)$ - by points. In Fig. 2a the useful signal is described by differentiated function with two local maxima, in Fig. $2 b$ - continuous nondifferentiable function and in Fig. 2c - discontinuous function. As basic functions $\left\{\varphi_{n}(x)\right\}$ Legendre polynomials (1) were used. The number of coefficients of the generalized spectrum used at restoration was chosen based on the condition $\langle\delta\rangle \leq 0.05$ (24) for the "worst" type of signal. As "worst" the signal for which the coefficients of the generalized spectrum decrease more slowly with increasing sequence number is meant (as follows from Table 1 this type of signal will be discontinuous signal). Thereby the $\mathrm{K}$ value made $K=15$ (it may be noted that generally speaking $K<K \leq_{\text {opt }}$ for the considered examples).

From Fig. 2 follows that the proposed algorithm (22), (23) allows to restore information signals against uncorrelated hindrances at input SNRs' values of the order or less $-3 \mathrm{~dB}$ to a high accuracy. The conducted additional researches have shown that the mentioned algorithm can be also used for effective extraction of signals against stationary Gaussian and Laplace hindrances (including hindrances with high enough intensity).

\section{Conclusion}

In this work the restoration algorithms of signals and images on the basis of their representation in the form of a set of coefficients of the generalized spectrum at absence and presence of random

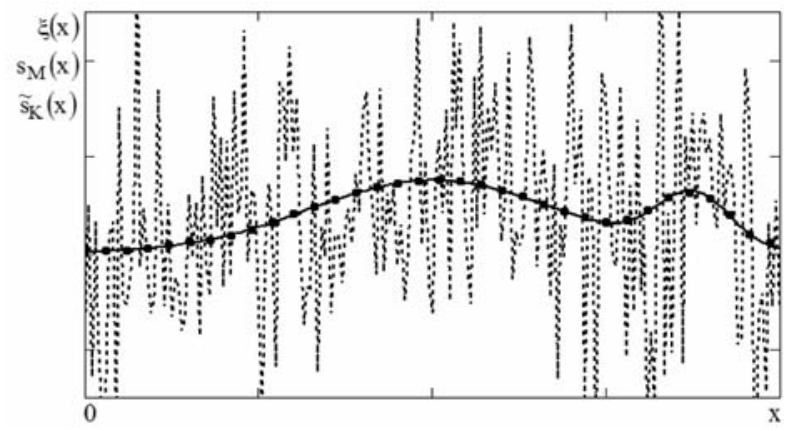

a)

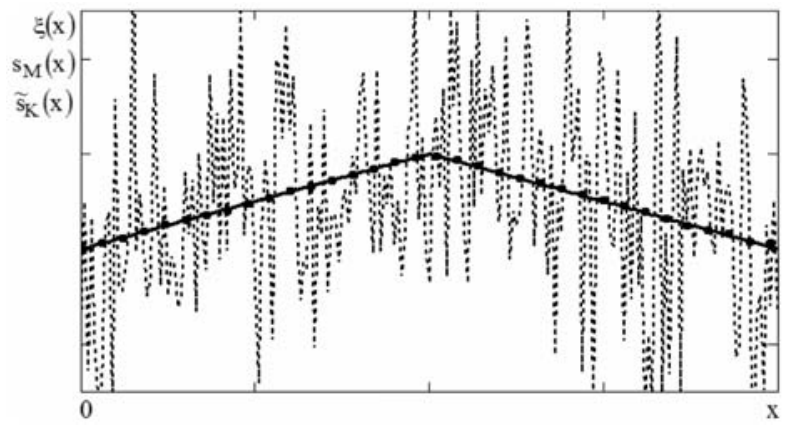

b)

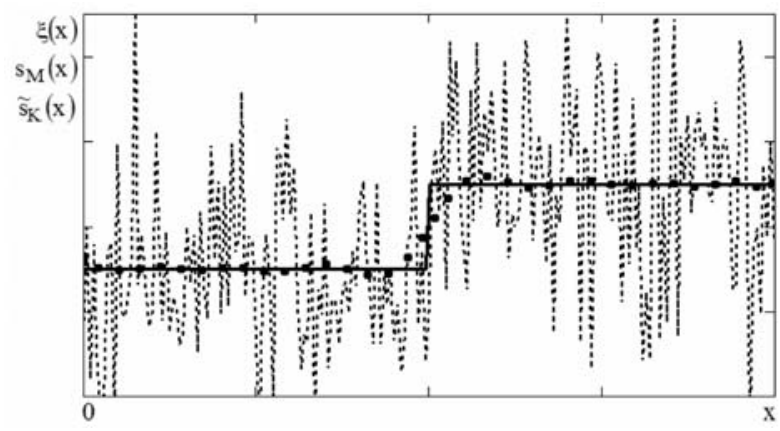

c)

Fig. 2 Original, noisy and restored signals with various behavior

distortions were considered. It was shown that the number of coefficients of the generalized spectrum of a restored deterministic signal (image) is defined by the desired approximation error at use of one or another metrics of functional space. Herewith at processing of continuous signals the application of Hermite polynomials or functions appears the most effective. If the useful signal is discontinuous then the construction of the generalized spectrum in basis of Chebyshev polynomials is more expedient.

In the presence of stationary hindrances the maximum-likelihood approach in aggregate with the generalized spectral signal 
representation in bases of orthogonal polynomials (functions) allows to receive the restoration algorithm of signals (images) which is rather effective (including the action of hindrances of enough high intensity), requires the minimum amount of the prior information (in particular, the form and other characteristics of a useful signal can be unknown) and can be practically realized in both analogue and digital variants sufficiently simply. Besides, in the general case there is an optimum number of coefficients of the generalized spectrum (relatively small because of fast decrease of the generalized spectrum with increasing the number of coefficient) required for restoration of a useful signal against hindrances here.
The results obtained allow to recommend the proposed algorithms for restoration of information signals and images which are deterministic or distorted by stationary hindrances (including correlated and non-Gaussian) in various practical applications.

\section{Acknowledgement}

The reported study was supported by Russian Foundation for Basic Research, research projects No. 12-01-09406-mob_z, 13-0800735a, and by The Ministry of education and science of Russian Federation, projects 14.B37.21.2102, 14.B37.21.2032, 14.B37.21.2015.

\section{References}

[1] VATOLIN, D., RATUSHNYAK, A., SMIRNOV, M., YUKIN, V.: Methods of Data Compression. The Archivers' Structure, Image and Video Compression [in Russian], Dialog-MIFI, Moscow, 2003.

[2] Digital Processing of Computer and Television Images, Edited by Zubarev, Y. B., Dvorkovich, V. P. [in Russian], Moscow Centre of the Scientific and Technical Information, Moscow, 1997.

[3] CHERNOYAROV, O.V., BREZNAN, M.: Optimal and Quasioptimal Algorithms of Distinction of the Compressed Images in Bases of Orthogonal Polynomials, Communications - Scientific Letters of the University of Zilina, vol. 14, No. 2, 2012, pp. 22-26.

[4] ChERNOYAROV, O. V., BREZNAN, M., GOLOBORODKO, P. A.: The Comparative Analysis of Optimal Estimations of Images' Shift Parameter in Synchronous and Asynchronous Orthogonal Function Bases [in Russian], Herald of the Moscow Power Engineering Institute, No. 3, 2012, pp. 103-109.

[5] SZEGO, G.: Orthogonal Polynomials, American Mathematical Society, 4 edition, 1975.

[6] HAZEWINKEL, MICHIEL: Encyclopedia of Mathematics, Springer, 2002.

[7] GULDAN, V., MARCOKOVA, M. Orthogonal Polynomials and Related Special Functions Applied in Geosciences and Engineering Computations, Communications - Scientific Letters of the University of Zilina, vol. 12, No. 1, 2010, pp. 12-15.

[8] RICHARD, L. BURDEN, J., DOUGLAS, FAIRES: Numerical Analysis, Thomson Brooks/Cole, 2005.

[9] Adaptation in Information Optical Systems, Edited by N. D. Ustinov [in Russian], Radio i Svyaz', Moscow, 1984.

[10] The Theory of Coherent Images, Edited by N. D. Ustinov [in Russian], Radio i Svyaz', Moscow, 1987.

[11] Systems of Machine Vision, Edited by Pisarevsky, A. N., Chernyahovsky, A. F. [in Russian], Mashinostroienie, Leningrad, 1988.

[12] TRIFONOV, A. P., SHINAKOV, Y. S.: Joint Discrimination of Signals and Estimation of their Parameters against Background [in Russian], Radio i Svyaz', Moscow, 1986.

[13] PRATT, W. K.: Digital Image Processing, John Wiley \& Sons, Inc., Hoboken : New Jersey, 2007. 